\title{
Erratum to: Glutamate receptors of the delta family are widely expressed in the adult brain
}

\author{
Régine Hepp - Y. Audrey Hay $\cdot$ Carolina Aguado $\cdot$ Rafael Lujan • \\ Luce Dauphinot $\cdot$ Marie Claude Potier - Shinobu Nomura • Odile Poirel · \\ Salah El Mestikawy $\cdot$ Bertrand Lambolez $\cdot$ Ludovic Tricoire
}

Published online: 20 August 2014

(C) Springer-Verlag Berlin Heidelberg 2014

\section{Erratum to: Brain Struct Funct \\ DOI 10.1007/s00429-014-0827-4}

The original version was incorrectly published as the given and family name of the author, Y. Audrey Hay was swapped. Y. Audrey is the first name and Hay is the last name of the author. The correct author name and her affiliation part are given below.

\section{Y. A. Hay}

Institut National de la Santé et de la Recherche Médicale (INSERM), U1130, Paris, France

The online version of the original article can be found under doi:10.1007/s00429-014-827-4.

R. Hepp · Y. A. Hay · S. Nomura · O. Poirel .

S. El Mestikawy · B. Lambolez $\cdot$ L. Tricoire $(\bowtie)$

Sorbonne Universités, UPMC Univ Paris 06, UM CR 18,

Neuroscience Paris Seine, 75005 Paris, France

e-mail: ludovic.tricoire@snv.jussieu.fr

R. Hepp · Y. A. Hay · S. Nomura · O. Poirel ·

S. El Mestikawy · B. Lambolez $\cdot$ L. Tricoire

Centre National de la Recherche Scientifique (CNRS), UMR

8246, Paris, France

R. Hepp · Y. A. Hay · S. Nomura · O. Poirel ·

S. El Mestikawy · B. Lambolez · L. Tricoire

Institut National de la Santé et de la Recherche Médicale

(INSERM), U1130, Paris, France
C. Aguado · R. Lujan

Departamento de Ciencias Médicas, Facultad de Medicina, Instituto de Investigación en Discapacidades Neurológicas (IDINE), Universidad Castilla-La Mancha, Campus Biosanitario, C/Almansa 14, 02008 Albacete, Spain

L. Dauphinot $\cdot$ M. C. Potier

Sorbonne Universités, UPMC Univ Paris 06, Inserm, CNRS, UM 75, U1127, UMR 7225, ICM, 75013 Paris, France

\section{S. El Mestikawy}

Department of Psychiatry, Douglas Hospital Research Center, McGill University, Montreal, Quebec H4H 1R3, Canada 